\title{
Créer pour recréer le lien avec l'environnement
}

\section{Edith Planche}

\section{OpenEdition}

\section{Journals}

Édition électronique

URL : http://journals.openedition.org/ere/2674

DOI : 10.4000 /ere. 2674

ISSN : 2561-2271

\section{Éditeur}

Centr'ERE

\section{Référence électronique}

Edith Planche, "Créer pour recréer le lien avec l'environnement », Éducation relative à l'environnement [En ligne], Volume 14 - 1 | 2017, mis en ligne le 15 septembre 2017, consulté le 21 février 2020. URL : http://journals.openedition.org/ere/2674 ; DOI : 10.4000/ere.2674

Ce document a été généré automatiquement le 21 février 2020. 


\title{
Créer pour recréer le lien avec l'environnement
}

\author{
Edith Planche
}

Ethnologue de formation, j'ai travaillé sur les positions d'énonciation autour de la scientificité, comme cristallisation de résistance du "sujet» au sensible et plus largement au domaine de l'image symbole et non pas signe. Quand bien-même le XXIe siècle scientifique voit les curseurs se déplacer entre «objectif » et "subjectif », ma recherche mettait en évidence les résistances de l'épistémologie rationaliste dans la manière européenne d'organiser le réel. Ainsi, le cloisonnement entre "Science » et «Art» qui nous coupe du symbole (soit du volume symbolique qui enrichit la vie pour enchanter le monde) et la rupture entre « Nature » et «Culture » qui nous coupe de nos ancrages du sol (de la nature, de la terre et des savoirs locaux) et sépare l'humain de son environnement, sont les deux interfaces d'un même clivage. L'association Science et Art a été créée pour tenter de réconcilier ces pôles, en particulier au sein de projets de sensibilisation au territoire en partenariat avec des collectivités territoriales ${ }^{1}$. Cette structure, qui s'inscrit dans le champ de l'éducation à l'environnement en France, remet l'art et la créativité (la Culture), mais aussi l'anthropologie (les cultures), au cœur de son approche. Les ateliers mis en place auprès de publics variés visent à rétablir un lien sensible avec l'environnement, qu'il soit culturel (mémoire d'un quartier, d'un territoire) ou naturel (faune, flore, paysage). Sur un plan éducatif, la démarche artistique invite à créer pour s'approprier, aimer et avoir envie de préserver son milieu de vie, tandis que l'approche ethnologique établit de la filiation en donnant $\mathrm{du}$ sens et du poids au territoire pour favoriser le désir de le protéger. Alors que l'ethnologie met au jour le faisceau de prolongements symboliques que l'habitant tisse avec la mémoire et son lieu de vie, l'art et la poésie permettent d'activer une forme de pensée reliée, à l'image de la "pensée sauvage ", une expérience augmentée par la voie du symbole qui tisse des connexions avec les objets du monde. L'anthropogéographe Jean Malaurie déplore un manque de réflexion profonde sur le clivage entre les peuples «racines» qu'il a fréquentés et les sociétés modernes: manque de ponts et de compréhension de ce que les formes de pensée, comme la "pensée sauvage", 
pourraient apporter au monde. L'art et la poésie sont des voies exotériques qui peuvent nous mettre sur le chemin de cette reliance avec l'environnement. ${ }^{2}$

\section{Pensée sauvage et pensée rationnelle, deux voies pour organiser le réel}

Claude Levi Strauss définit deux grands modes de pensée pour appréhender le réel.

[La pensée rationnelle] est déterminée par l'intelligible: nous évacuons nos sensations pour manipuler des concepts. À l'inverse, la pensée sauvage calcule, non pas avec des données abstraites, mais avec l'enseignement de l'expérience sensible : odeurs, textures, couleurs. Dans les deux cas, l'homme s'emploie à déchiffrer l'Univers, et la pensée sauvage, à sa manière, y parvient aussi bien que la pensée moderne. ${ }^{3}$ (Cité par Sorman, G., 1989).

Ces deux formes de penser le monde sont accessibles à chacun et chaque peuple peut $\mathrm{y}$ prétendre. Alors que la rationalité exclut tous les sens, le savoir-faire du peuple " racine », à l'écoute des éléments, ré-introduit tous les sens, à l'image de nos savoirfaire du terroir qui favorisent la sensualité. Ainsi, le fromager jurassien nous dit que son rapport au comté est un peu «fusionnel » et que le fromage parle ${ }^{4}$. C'est en effet à partir de sa sensation - en «écoutant » la meule (sons), en la tapotant (touchers), en la humant (odeurs) - qu'il oriente ses gestes pour passer à un autre stade de l'affinage, tandis que l'industrialisation proposera une machine à mesurer, appliquée à toute la cave. La tendance dominante de la rationalité occidentale consiste à tout séparer en deux, nous dit François Laplantine ${ }^{5}$ : la raison et les sens ; l'objectif et le subjectif; les choses, le minéral et les êtres vivants; les animaux et l'humanité. Alors que dans la pensée animiste au sens générique, tout est relié, le soubassement culturel de nos sociétés européennes s'évertue à défaire les liens pour forger des «idées claires et distinctes » :

Un Canaque lui dit [à l'ethnologue et pasteur Maurice Leenhardt], à son grand étonnement, que c'était le christianisme qui lui avait appris qu'il avait un corps. Un corps, c'est-à-dire d'abord un objet qui était séparé de l'âme, au lieu d'engluer l'esprit dans la matière - un corps, c'est-à-dire aussi un objet séparé des autres objets de la nature, des pierres totémiques, des animaux et des végétaux, comme du nom qu'on lui donne, de toutes ses appartenances. (Bastide, 1965, p. 137-156)

La pensée rationnelle a ses avantages: elle met de l'explication là où il $\mathrm{y}$ avait « confusion» (au sens propre de choses mêlées). Elle sépare les données pour donner une lecture "verticale». Elle a tendance à diviser, à spécialiser de manière unidisciplinaire en isolant ou en excluant les sens, les sujets qui pourraient être associés ou apparentés, tout ce qui peut parasiter la réflexion et l'expérience comme le débordement affectif ou la subjectivité de l'acteur. L'ethnologue Roger Bastide nous montre comment le Cartésianisme ${ }^{6}$ a influencé tous nos modes de pensée modernes en nous invitant à brider les égarements sensibles :

Descartes fait de la philosophie une méditation sur la cogitatio : "les corps ne sont pas proprement connus par les sens, mais par le seul entendement”, c'est-à-dire qu'en tant qu'idées claires et distinctes. Son idéal reste celui des mathématiques et il n'a de cesse que de modeler toutes les pensées sur le modèle des essences mathématiques. Il sait cependant que pour y arriver, il faut être "fort attentifs" et détacher son esprit "du commerce des sens". C'est que son analyse de la cogitatio lui fait reconnaître, au dedans de l'esprit, à côté des idées claires et distinctes, une 
pensée obscure et confuse, pour employer ses propres termes ; obscure c'est-à-dire non analysée, et confuse. (Bastide, 1965, p. 137-156) technique : pour atteindre «l'objectivité » et observer le réel, la pensée rationnelle se doit d'exclure le sujet, de séparer l'être humain des objets du monde et de l'ensemble du vivant. alternance ou une mixité, des formes de pensée complémentaires :

- Une pensée verticale qui creuse, se spécialise et analyse - modèle universel et formaté «tout sol» (qui peut s'appliquer partout), détaché du contexte local, abstrait - ET une pensée horizontale qui relie, qui introduit les sens, lie les savoir-faire et les savoir-être. ${ }^{8}$

- Une pensée de la catégorie qui sépare et divise pour mieux observer ET une pensée de l'énergie qui rythme, une pensée symbolique qui tisse des toiles entre les réalités pour saisir le réel (domaine de l'intuition, du chaman, de la spiritualité, de la transcendance, de l'art et de la poésie). L'art peut être un prétexte exotérique pour changer de catégorie logique. Roger Bastide voyait émerger dans la transe une approche de "la pensée de l'énergie » susceptible de subvertir et de submerger «la pensée de la catégorie", nous rapporte François Laplantine : «pour pouvoir comprendre les cultes du candomblé [...] il me fallut, écrit-il, changer de catégories logiques ». (Laplantine, 2005, p. 134, p. 137). Pensée en image et sensorielle, la poésie, comme le candomblé, semble faire appel à d'autres véhicules que la rationalité, pour s'approcher d'une pensée plus globale, plus intuitive, plus complète.

\section{Recréer le lien avec l'environnement : De la pensée systémique à la « pensée sauvage »}

7 Le mouvement de l'écologie profonde met en exergue les risques de dérives d'une conception du monde où la perte du lien sensible avec l'environnement nous conduit à penser l'être humain comme pouvant «jouer » sans la nature considérée alors comme accessoire. Les sociétés traditionnelles ne sont pas a priori des sociétés modèles. Néanmoins, elles cultivent le dépassement de cette pensée rationnelle qui coupe l'humain de la nature et de son lien au monde. La rationalisation rompt les liens, car elle offre une réponse généraliste, détachée du contexte local, qui va s'appliquer pour tout et pour tous. C'est toute la logique de la rationalisation de la vie et du travail hors sol, à l'opposé d'un mode de vie et de penser qui relie le travail, les valeurs de la communauté et la nature de proximité. L'exemple type de la rationalisation extrême nous est montré par la soviétisation des peuples autochtones du Nord de la Sibérie. La vie de l'éleveur de rennes épousait les mouvements de la toundra guidant les animaux, forgeant des liens entre les valeurs de la communauté et l'écoute des signes de la nature assurant sa survie. La soviétisation a engendré la rationalisation de l'élevage s'adaptant à des règles définies a priori. Malgré un effet secondaire positif avec l'unification vertueuse des ethnies, les nomades ont été contraints à la sédentarisation, les troupeaux de rennes collectivisés tandis que leurs propriétaires intégraient les kolkhozes et les sovkhozes comme hommes-ouvriers, le système entravant ainsi les chemins de traverse jusqu'ici calés sur les rythmes de la nature.

Marc Tadié nous montre l'impact dans le cerveau, de la distanciation de tous les sens qui ne conditionnent plus la survie de l'individu moderne. (Tadié, 2001, p. 31-53). A 
contrario, "se situer dans le milieu boréal, c'est devoir faire appel à tous ses sens " (Etiembre, 2016), lorsqu'il s'agit de silence et de survie...

Plus que vers ces invisibles sensibles du rapport sensoriel au territoire qui émergent dans l'artisanat et les modes de vie traditionnels (invisibles sensibles et sensoriels liés à l'importance du geste humain, aux affects de la transmission orale et/ou familiale, mais aussi à la correspondance entre les valeurs, les activités, la vie et le sol), les sociétés en prise directe avec la nature vont-t-elles en fin de compte nous guider vers la voie de la communication avec les invisibles ou la «préscience sauvage " évoquée par Jean Malaurie lorsqu'il rend hommage aux Inuits, ces méditants contemplatifs qui lui ont appris le « silence » ? (Planche, à paraitre 2019)

En effet, l'archétype des peuples premiers se relie à l'environnement via l'approche sensorielle de la nature qui le "nature", le met au cœur des correspondances écosystémiques pour accéder à d'autres entendements. Les cultures orales ne font pas de la poésie comme Rimbaud', mais leur extrême connexion avec l'environnement implique cette perception des liens entre les éléments ${ }^{10}$, à la manière du poète, comme l'exprime Jules Supervielle. Pour lui, le poète voit les liens invisibles qui s'enchevêtrent dans notre corps, entre nous et tous les humains et les animaux (cité par Planche, 2008, p. 14). Il est donc bien question de liens. A l'image de la "pensée sauvage » ou de la pensée animiste, le langage poétique tisse de subtiles images entre les choses du monde, à travers la dynamique des mots et des sons, qui produisent de l'image, mais aussi de l'énergie; de même, le langage de la peinture tresse des fils de résonnances, entre la couleur, les volumes et les formes pour capter l'environnement.

Selon la théorie de Georges Devereux, toute société privilégie une » matrice primaire » à l'interface d'une «matrice secondaire » ou refoulée. Le poète ou l'artiste sont ainsi les gardiens, dans nos sociétés technico-industrielles, de la vitalité de la pensée ronde de l'analogie, qui n'est pas celle de la pensée carrée, objective, technique et froide ${ }^{11}$, mais celle de la "pensée sauvage» ou, comme le dit François Terrasson, celle du monde invisible de la sibylle, "cette mystérieuse entité tapie dans l'obscurité qui parle le langage de la nature et utilise un moyen de communication construit non avec des mots mais avec des symboles ». (Terrasson, 1997, p. 39).

\section{Entrer dans la posture et la démarche du poète ou de l'artiste pour « remettre des esprits dans la forêt » et se relier à l'environnement}

11 Se mettre à l'écoute de la résonance des couleurs comme l'artiste, ou développer la perception sensible de son environnement (air, humidité, sons, vent) comme le poète, permet de s'extraire de la classe, des cases, des classifications, pour faire jouer la "pensée sauvage», cette intelligence que l'Inuit possédait, "ravivée par l'enseignement des mythes, [...], un chuchotement des pierres, un bruissement " (Malaurie, 2015, p. 102). Aussi, le poète, comme l'animiste, se mettra à l'écoute des pierres, des animaux et du vent ...: « Écoute plus souvent / Les choses que les êtres / La voix du feu s'entend/ Entends la voix de l'Eau / Écoute dans le vent / Le buisson en sanglots : C'est le souffle des ancêtres » murmure Birago Diop, poète africain. 


\section{De la « pensée sauvage » à la poésie}

12 A l'image du Sarayaku, qui évoque la "forêt vivante $»^{12}$, l'animiste ne voit pas avec les contours profanes (utilité, appropriation mercantile, fonctions pour l'Homme), mais avec le «cœur" qui fait silence sur le désir de capturer et de maîtriser, avec une conception sacrée qui conçoit les éléments de la forêt dans leur paradigme autonome de vie. On considère ici les éléments de la nature comme animés par l'esprit de vie, dans leur identité de sujet avec lequel communier, composer, entrer en rapport de force ou d'alliance.

Tandis que les premières nations ont intégré les lieux de science pour prendre en main leur destin, tout en se reliant à leur culture ancestrale, la posture du poète autorise l'expression du côté "animiste» des sociétés modernes. La poésie nous invite à remettre des esprits dans la forêt, comme le préconisait François Terrasson, soit réinjecter du symbole, du lien de réciprocité et d'amitié. Il s'agit ainsi pour l'artiste, d'opérer un renversement de paradigme lorsque ce n'est plus nous qui regardons la forêt, mais la forêt qui nous pénètre et nous appelle. » Dans une forêt, j'ai senti à plusieurs reprises que ce n'étais pas moi qui regardais la forêt. J'ai senti, certains jours, que c'étaient les arbres qui me regardaient, qui me parlaient", nous dit André Marchand cité par François Laplantine. Ce dernier rappelle que «L'un des plus grands intérêts de la peinture - en particulier depuis Paul Klee - est de nous montrer qu'entre l'œil et le monde, les rôles tendent à tout moment à basculer." (Laplantine, 1996, p. 74-75). Ainsi, en est-il de la Balade poétique (Planche, 2008) proposée lors de nos activités d'éducation à l'environnement par le sensible: cette ballade invite à l'émerveillement en exaltant nos sens, nos souvenirs et nos sensations les plus intimes, les plus subjectives au contact de l'environnement. Dans un mouvement inverse, elle nous incite à faire taire toutes nos pensées, pour mieux contempler. Alors, à la manière de Rimbaud qui parlait de sa poésie comme objective, nous surferons sur la vague de l'entre deux, du silence bruyant, les yeux à demi clos pour écouter et capter le monde. Nous laisserons affleurer les images, afin de retisser du lien, de remettre du symbole, de réenchanter notre rapport au monde. Laissons-nous happer et guider par l'herbe, l'eau, le vent pour favoriser l'écoute et donner de l'espace au monde "invisible» du lien secret avec la nature. Prenons la posture du poète pour donner la parole à la nature. La nature nous le rendra bien et nous donnera la main pour écrire. Nous tisserons alors des toiles de mots et de sensations pour attraper l'indicible de notre relation à la nature.

\section{La posture animiste au regard de la posture « naturaliste »}

Philippe Descola ${ }^{13}$ est visionnaire lorsqu'il présente les sociétés animistes par opposition aux sociétés «naturalistes", définissant ces dernières comme eurocentriques, mais aussi comme anthropogéniques. Cet anthropologue établit une classification des cultures en créant un jeu entre identités et différences des intériorités et extériorités, qui oppose radicalement le "naturalisme » européen à "l'animisme ». Selon ce modèle idéal-typique, pour la posture "naturaliste" issue de la pensée moderne, l'humain et les autres vivants se rejoignent dans une identité commune qui se situe au plan de leur "extériorité ", c'est-à-dire de leur nature biologique (ce qu'il distingue comme "extériorité semblable", soit fait de la même matière biologique). Par contre, ils se différencieraient par leur intériorité, seul l'humanité parmi les vivants 
possédant une « âme » ou une « conscience » ou - exprimé de façon plus scientifique une capacité à penser et à élaborer en deçà d'une mécanique biologique. Au contraire, dans une société animiste, humains et autres existants se rassemblent et se reconnaissent par une "intériorité semblable ", c'est-à-dire une capacité à abriter une âme, ou - dit de façon plus scientifique - à être animé par l'étincelle du vivant et un projet de vivant. Par contre, la posture animiste différencie chaque existant par son extériorité, soit la forme de ses appareillages, qui correspond à son mode de vie et détermine sa différence de point de vue sur le monde. Dans le monde animiste, animaux, mais aussi minéraux, plantes et lieux peuvent être animés d'intentions. Et l'être humain va établir des relations de dialogue avec le milieu en essayant d'atteindre ces intentions, en se décentrant de la posture d'humain pour épouser le point de vue de l'autre, ne serait-ce qu'à travers les incorporations sorcellaires ${ }^{14}$. Pour exemple, l'animiste amérindien peut se demander si cet homme si agile et fort ne serait pas un jaguar déguisé en humain ou si cet oiseau qui pépie sur l'arbre ne cacherait pas un humain qui cherche à l'épier ou à le calomnier. On peut schématiser le modèle occidental en forme de pyramide, avec l'humanité au sommet du vivant, tandis que le modèle animiste se manifeste par une forme circulaire ${ }^{15}$ où chaque point de vue est entier et recevable sur le théâtre du vivant.

Au premier abord, le terme d'extériorité pour qualifier le lien entre les êtres vivants par leur nature biologique, peut apparaître comme inapproprié puisqu'il fait référence à la nature chimique du vivant et donc à sa constitution. Or, c'est bien d'une extériorité dont il s'agit par opposition à l'intériorité et l'insaisissable du «cœur de la raison d'être ", de la conscience de vie, de l'étincelle du vivant, du " conatus ", "cet effort que déploie tout organisme vivant pour persévérer dans son être et accroitre sa vitalité.» (Spinoza cité par Lenoir, 2015, p. 109). C'est cette vision animiste du monde que nous essayons de saisir à travers les décrochages de la pensée rationnelle que provoquent les espaces de respiration poétique. Mais pour cela, il est nécessaire de s'extraire du regard du savant qui observe son environnement de manière fonctionnelle. La séquestration dans le regard qui voudrait contenir un savoir nous sépare de l'observé. Le regard libre initie à cet enchantement de tout ce qui marche ensemble et s'éveille, chante à la vie. Il invite à l'accueil de la symphonie du monde, à l'acceptation de l'autre tel qu'il est avec ses intentions traduites par ses organes perceptifs, à la saisie de la réalité extérieure à nous -notre environnement - pour aller dans le sens de Goethe.

Que signifie commercer avec la nature, s'interroge Goethe dans La métamorphose des plantes, si nous n'avons affaire, par la voie analytique, qu'à ses parties matérielles, si nous ne percevons pas la respiration de l'esprit qui donne un sens à chaque partie et corrige ou sanctionne chaque écart par une loi tout intérieure? (Goethe, cité par Malaurie, 2001, p. 113)

Ainsi, l'animisme ou le chamanisme et leurs tentatives de rencontrer d'autres mondes à travers la communication avec le vivant ou l'incorporation des points de vue des animaux, seraient-ils proches de nos découvertes éthologiques qui nous montrent que chaque espèce avec son Umwelt, évolue dans une réalité de perception différente, comme dans des mondes parallèles?

Une prairie offre-t-elle aux yeux d'un taureau, d'une mouette ou d'un rotifère le même aspect qu'aux nôtres? Selon la théorie des milieux de Uexküll, chaque animal vit dans un univers propre reflétant le vécu de son organisation sensorimotrice (son Umwelt). (Clément, 1999, p. 20) 
17 A l'image du chaman qui se glisse dans les intentions animales, nous invitons l'apprenant, via la posture du poète, à rencontrer la faune ou la flore dans leur nature déployée, en faisant silence sur les explications, pour rentrer en amitié avec leur raison d'être, pour recréer le lien. "La rêverie poétique "sympathise" intimement avec le réel, tandis que l'approche scientifique est "antipathique" : elle prend ses distances avec la charge affective du réel » nous dit Bachelard.

Recevoir cet animal qui fait sa vie, se mettre à son échelle de besogne, c'est l'accueillir dans sa réalité d'être vivant. [...] Il s'agit de ne plus avoir envie de piétiner depuis cette hauteur d'Homme, mais de se mettre tout bas, dans l'herbe, et de regarder circuler ce vivant. Il bouge, il est en mouvement, il fait sa vie dans sa solitude d'être... Je ne rentre plus en relation avec mon air supérieur qui « sait ». Je m'implique. J'écoute l'autre - à égalité - : le renard, la belette, l'insecte,... l'araignée qui me fait peur car elle va très vite mais que j'admire lorsqu'elle saute, fait la morte, courre dans la multiplication des pattes et endort sa proie en l'anesthésiant. Je reconnais cet Autre vivant - tel qu'il est - en dehors de sa carte d'identité d'animal classé. (Planche, 2018, p. 188-189)

L'exercice poétique est au fond familier de la pensée animiste, lorsque le chasseur se met dans la peau de sa proie : «Et parce que pour prévoir les réactions de la baleine l'homme doit un peu devenir baleine lui-même, il va exécuter une gestuelle mimant les attitudes de la baleine afin d'intégrer l'esprit du cétacé » (Tadié, 2001, p. 43).

\section{La subjectivité et ses résonances ou recréer le lien avec l'environnement}

Recréer des liens sensibles avec l'environnement, c'est aussi favoriser le lien entre le sujet perçu (l'environnement) et soi-même, en étant attentif à une évocation personnelle, une impression en surimpression, une vision déformée, une humeur ou une frayeur en laissant de la place au subjectif, aux résonnances émotives plutôt qu'aux raisonnements. Je reste attentif à mes sensations, à mes sentiments, aux pistes qui s'ouvrent. Je me heurte à un petit caillou, je le ramasse, il a des reflets roses comme la Provence de mon enfance. L'accroche sensible et émotionnelle va déclencher les souvenirs : c'est le principe de la madeleine de Proust. Pour remettre du lien avec le paysage, on propose par exemple de recenser sur la page de gauche tout ce qui s'inscrit dans son champ de vision, tandis que la page de droite fait la ligne de démarcation pour laisser courir sa pensée en associations d'idées et de mémoire. Il s'agit de porter son attention sur la manière dont on associe constamment son environnement « objectif » à du vécu et des impressions subjectives, afin de s'en servir pour alimenter un réservoir d'idées poétiques et/ou de créer un attachement qui redonne du sens. Nous pourrons représenter ce " poids" des perceptions qui nous soutiennent, en tant que miroir de nos projections, en prenant modèle sur l'œuvre de Peter Doig intitulée «Reflection » (What Does Your Soul Look Like ?). ${ }^{16}$ Ce tableau est constitué en effet de tout ce qui se passe en dessous de l'Homme : deux jambes apparaissent au-dessus d'un miroir d'eau où se reflète un monde souterrain, riche et vivant. Nous inviterons chacun, en s'inspirant de ce tableau, à illustrer ses découvertes émotionnelles et les mouvements intérieurs de sa pensée au contact de l'environnement en peignant le socle de ses perceptions. Ce sera également l'occasion d'apprendre à représenter les reflets d'eau.

20 La sortie plein air nous relie à l'environnement parce que nous sollicitons l'attention « ouverte», pour que l'esprit soit distrait, happé par l'extérieur, par les images qui 
génèrent des images, pour pénétrer dans ce monde d'images... et développer la créativité, donc la bonne santé, la vivifiance de l'esprit.

Que le but final soit la créativité ou la science, on aura laissé un espace pour cette "dérive", en cadrant des temps pour cela, pour passer ensuite à la phase de " maitrise ", où l'on va ordonner le réel, déterminer la nature de cette pierre rose ou se servir de son panier d'images pour mettre en forme une création.

\section{Jouer avec le paysage pour « rentrer » dans le paysage}

Il faudrait non seulement avoir des connaissances, mais aussi des sentiments d'appartenance à la planète Terre, déclarait François Terrasson. ${ }^{17}$ Aussi, quand les sociétés paysannes développent une attention aux saisons et aux correspondances écosystémiques, quand le "peuple racine" résonne avec la nature et échange de l'énergie avec elle, quand il ne se sépare pas du paysage qu'il conçoit comme investi par des forces et des ancêtres, l'environnement n'est pas conçu comme un épiphénomène, mais il se situe comme élément de la nature avec laquelle l'humain joue sa vie. "Remettre des esprits dans la forêt ", c'est aussi prendre contact avec le paysage en y mettant du sens et donc des "forces" d'investissement, pour "transformer nos rapports d'usages en rapports du sage », comme l'évoque Gaston Pineau (1992, p. 237).

La création permet de recréer le lien avec l'environnement : elle autorise à « habiter » le paysage avec une partie de soi-même (ses projections subjectives, ses choix, le déclenchement de l'imaginaire), elle tisse des tentacules entre ses propres visions et l'environnement. Nous rejoignons l'étymologie des noms de lieux qui puisent souvent leurs origines dans des repères imagés (comme La dent du chat, Le bec de l'Aigle). Un atelier de lecture de paysage va par exemple nous autoriser à laisser parler notre imagination: on va essayer de voir des formes, d'exagérer ces erreurs de vision pour habiter le paysage avec ses représentations. On fréquente ainsi l'animisme par le dessin en recherchant un autre regard, à l'image de l'œuvre de Magritte » Le domaine d'Arnheim (1962) ", où le sommet de la montagne prend le visage d'un aigle ou bien de façon plus complexe, à la manière d'Arcimboldo lorsqu'il articule un pont et une montagne pour faire surgir un visage dans le paysage. On se référera au thème de l'Homme-paysage en peinture dans l'art occidental, avec des artistes de l'École du Nord comme Matthäus Merian (Paysage anthropomorphe, Aquarelle vers 1650), Joos de Momper II (Allégorie de l'automne, XVIIe siècle) ou Le portrait de femme par un Anonyme (Musée Royal des Beaux-Arts de Belgique, XVIe siècle). Il sera fructueux d'appliquer ce concept artistique à un paysage familier, ce qui permet de le rendre vivant, de réinjecter du volume et du lien, avant de rebondir sur l'analyse géographique.

On s'intéressera aux erreurs de vision en plissant les yeux, en changeant d'angle de vue, voire en multipliant les angles, à la manière de Picasso et des cubistes. Consulter et fréquenter des œuvres ouvre l'imaginaire des formes (plus que l'imagination). On peut retracer les grandes lignes et masses du paysage, sans s'attacher au détail, puis y replacer l'élément transformé (une colline devient un chapeau, le pont ressemble à un chameau, cet arbre à un personnage à figure humaine $)^{18}$.

Alors que les sociétés traditionnelles conçoivent des paysages habités par des forces et des ancêtres, nous évoquerons, quant à nous, les paysages internes avec nos accroches sensibles au territoire, qui nous permettent de nous attacher au paysage. On proposera, par exemple, de représenter le paysage de façon plus abstraite, comme condensé de 
symboles et de repères de la vie personnelle, en le composant uniquement de références subjectives. Il s'agit de se laisser guider par la mémoire ou les associations d'idées pour dessiner son paysage-souvenir. Les métaphores peuvent être plus ou moins poussées pour des effets artistiques. Cette rue symbolise pour l'adolescent que je suis l'objet de plaisir que je vais régulièrement y acheter - un $\mathrm{CD}$ de musique. Pour mon cousin, elle renverra à l'idée du dentiste qui a son cabinet au 10 de la rue : cette idée structurera un paysage un peu sanglant ou effrayant avec des objets médicaux qui apparaissent ça et là ou inspirent le design de quelques mobiliers urbains. Ou bien je représente la cueillette des murons sous la forme des mains de ma grand-mère cerclées par un cœur couleur mûre. On s'inspirera des productions de René Magritte : Bouteille peinte (deux hommes en conversation), 1942 ; Les valeurs personnelles (1952); La saveur des larmes (1948).

Si l'immersion dans la nature peut être très marquante parce qu'elle nous permet de décrocher du cérébral, la stimulation de l'empathie affective par la " poète attitude » et la démarche artistique est également plus profonde, car elle permet de décrocher du mental pour avoir accès à d'autres perceptions. Ce sont ces connexions sensorielles qui semblent recréer le lien avec les objets du monde, via les tentacules du symbole et des images.

\section{Plasticité de l'artiste et du chaman ou l'immersion dans la physis}

Pour le peintre Jean Dubuffet, «L'art ne vient pas coucher dans les lits qu'on a faits pour lui ; il se sauve aussitôt qu'on prononce son nom : ce qu'il aime c'est l'incognito. Ses meilleurs moments sont quand il oublie comment il s'appelle». Ce n'est pas l'apparence des choses que l'artiste tente de saisir, mais l'invisible : ainsi, l'artiste trop bien peigné que Rimbaud fustige renvoie à un artiste qui cherche à créer au lieu de se laisser pénétrer, ou à une œuvre qui se pense plutôt qu'elle ne se laisse saisir: «Baudelaire est le premier voyant, roi des poètes, un vrai Dieu. Encore a-t-il vécu dans un milieu trop artiste » (Rimbaud, 1965). A l'image de la plasticité du chaman, l'artiste doit se délester de sa volonté dirigée. « On devrait dire : on me pense » (Rimbaud, 1965). Comme Rimbaud dénigre le poète trop bien intentionné et l'invite à saisir plutôt qu'à construire, l'Inuit évite toute cristallisation en laissant de l'espace au discours et à l'appel de la nature. Ainsi, ce dernier dit à Malaurie que trop penser figera son savoir et l'empêchera d'être un bon chasseur à l'écoute de son intuition. On saisit ici la proximité entre le peintre naturaliste - chasseur d'images - et le chasseur animiste, qui doivent faire silence en se délestant d'une volonté préconçue, pour se mettre en relation avec la nature et ses mouvements, et accéder à une perception inspirée qui est relation-union avec l'intention du sujet croqué ou avec l'intention de la proie à croquer.

\section{Communiquer avec les invisibles pour reconnecter avec sa pensée intuitive}

Selon Platon, l'exercice de la poésie va de pair avec une forme de dépossession : «Qui qui se sera, sans le délire des Muses, présenté aux portes de la Poésie avec la conviction que l'habileté doit, en fin de compte suffire à faire de lui un poète, celui-là est lui-même un poète manqué, comme est éclipsée par la poésie de ceux qui délirent celle de 
l'homme qui se possède! ». (Platon, Ceuvres complètes, tome 4, 3ème partie, Phèdre, texte établi et traduit par Léon Robin (1970). Paris : Les Belles Lettres, p. 32-33). Et tout le talent du poète ou de l'artiste véritable est de maintenir une attention perceptive, de garder cette porte ouverte à la multiplication des résonances et à la perception "pleine", de se laisser pénétrer par les images plutôt que de les inventer. Pour fréquenter ces espaces de l'inspiration, ces espaces intuitifs, il faut faire silence sur ses projections humaines ou ses intentions dirigées, pour se laisser guider, happer par l'environnement... Et nous rejoignons la pensée du chasseur qui sait suspendre le raisonnement afin de laisser le passage à son intuition perceptive. «Ils m'expliquent qu'écrire figera leurs forces d'intuition, qui seront, selon leur expression 'canalisées et presque normalisées' : 'je ne serais plus libre, m'a dit un très jeune Netsilik, et je serais un mauvais chasseur'« (Malaurie, 2015, p. 108-109). En effet, écrire et consigner, c'est figer le mouvement, cristalliser, fermer...

\section{Jouer avec l'image, déformer, transformer pour se relier à l'environnement}

De l'autre côté de l'épistémologie rationaliste, les peuples animistes, mais aussi l'artiste ou bien encore les écoformateurs ne nous proposent-ils pas paradoxalement de tendre vers une liberté de regard, loin des catégories de classification humaines, de quitter une extériorité observatrice, en « rentrant » dans l'univers du milieu, en s'impliquant au sens de Dominique Cottereau, qui oppose implicare, "se sentir dedans » à explicare, car pour sensibiliser, les explications et l'information ne suffisent pas. En effet, pour l'artiste Cézanne, le maître du regard, « Regarder ne signifie pas rester à l'extérieur de l'objet, le tenir rassemblé sous son regard mais sortir de soi, aller à la rencontre de l'objet, entrer en lui... » (Aurégan, 2015, p. 107).

L'éducation à l'environnement, par la connaissance naturaliste, nous demande souvent de classer, soit de capturer le monde dans le regard humain en se positionnant en surplomb. En quittant les contours de nos catégories, nous nous laissons plus facilement guider par la résonnance des formes, des couleurs, des objets qui s'animent, des insectes qui virevoltent. L'artiste adopte une attention «flottante» ou contemplative pour se mettre à l'écoute, entrer en amitié avec la nature, se situer à égalité de posture. A l'image de l'animiste, l'artiste Jean Arp rend son point de vue à la nature: "L'homme fait à tous les instants des déclarations définitives sur la vie, l'homme et l'art, et ne sait pas plus que le champignon ce qu'est la vie, l'homme et l'art ». Ce sculpteur préconise l'humilité devant la créativité de la nature qui donne forme. Avec Jean Arp, « il ne s'agit pas de parler de la nature ni de parler pour elle, mais de parler comme elle et par là, d'agir comme elle afin de la transformer et de se transformer avec elle, en elle.» (Arp, 1986, p. 61). On retrouve ici une proximité avec l'éco-formation où éduquer à l'environnement, c'est aussi reconnaître et éprouver cet aller-retour :

L'environnement forme au moins autant qu'il est formé ou déformé. Le terme écoformation veut traduire et explorer cette réciprocité [...]. Ce n'est qu'en sachant comment l'environnement met en forme que nous saurons comment former un environnement viable et durable. (Cottereau et Bachelart, 2003)

31 L'artiste doit produire en s'alignant sur la dynamique des formes de la nature et non reproduire ce qu'il voit. Avec Arp, il ne s'agit pas de s'inspirer d'une nature comme levier d'imagination, ni encore moins de copier la nature. L'artiste s'imprègne et 
s'immerge pour faire partie du processus de la nature qui le guidera dans ses gestes, à l'image de l'inuit qui sait s'abreuver au "lait» de son environnement (naturel, mais aussi spatial) qui devient informant et "déformant», impactant sur le mental et la pensée, pour l'alerter en retour. ${ }^{19}$

L'œuvre miroir peut agir comme révélateur de notre lien intrinsèque au monde L'imaginaire peut devenir galopant et nous emmener plus loin que nous-mêmes et que nos savoirs conscients. Ainsi, je vois des formes qui représentent des volumes, des vides et des pleins, rentrant quelque part dans cet univers de "forces ». La pensée artistique est une pensée en images qui invite à élargir, à circuler, à recycler. C'est une pensée nomade comme la pensée chamanique, capable de faire des liens entre les choses du monde que nous considérons ordinairement comme distinctes. Elle nous permet donc de saisir le réel autrement, de nous emmener ailleurs, hors des catégories logiques, à l'opposé des cadres distincts, assurés, tranchés, clairs et nets.

L'image nous permet-t-elle de nous déposséder de ce point de vue rationnel et unidirectionnel, pour appréhender le monde avec d'autres canaux de perception du réel ? Le poète surfe sur les mots s'en se fixer sur leur définition, pour contourner l'indéfinissable et prolonger le définissable car, comme le dit si bien l'Inuit qui préconise de se méfier des mots, «ils congèlent les pensées » (Malaurie, 2001, p. 139). Lorsqu'on pense avec des mots, on pose les cadres de nos limites, tandis que le poète pense avec des symboles (soit des mots-images multisignifiants), plus « ouverts » et connectés à la toile du monde. (Planche, à paraître 2019)

« [Aussi le langage] reste-t-il, ajoute Goethe, presque toujours à court, rabaisse son sujet ou même le mutile et... l'anéantit » (Malaurie cité par Aurégan, 2014, p. 162).

Le dépassement des frontières entre les objets et les sujets du monde ou une certaine forme de perméabilité caractérisent le chamanisme: «La personnalité déborde les limites du corps, pour s'étendre, comme des pseudopodes, sur les autres personnes du groupe, sur des pierres, des animaux, des végétaux en même temps que le groupe, la pierre, l'animal et le végétal entrent dans la personnalité. » (Bastide, 1965, p. 137-156.). Comme l'artiste qui se laisse entrainer par la résonnance de formes et de couleurs, le chamane fait une incursion dans ce monde de synchronicité, où les niveaux de réalité communiquent par le jeu des symboles, où les éléments se connectent dans une autre forme de logique. Le chamane comme l'artiste dépassent la perception commune et rentrent en connexion avec "le monde", surfant sur d'autres paradigmes. Et cette pensée non fixée nous parle de voyage lorsque les limites entre les mondes, entre les Hommes et les animaux, entre les lieux et les dieux, entre les objets, se déplacent, se forment et se déforment.

Cette démarcation entre physique et métaphysique est perméable, tout comme la frontière entre l'homme et l'animal. Ainsi les limites du monde humain sont-elles poreuses. Cette perméabilité est la façon de voir et de comprendre le monde qui forme la base du chamanisme. (Etiembre, 2015)

Jean Arp, à travers ses jeux de formes, peut nous familiariser avec cette forme de pensée nomade. L'artiste "met en place une grammaire des formes, "l'encyclopédie arpadienne". Il fait varier la représentation de ces formes dans des "rencontres fortuites" et les combine dans ses œuvres. » (Archives INA)

Souvent, un détail d'une de mes sculptures, un galbe, un contraste me séduit et devient le germe d'une nouvelle sculpture. J'accentue ce galbe, ce contraste, et cela entraîne la naissance de nouvelles formes... Il me faut souvent des mois, des années pour mener à bien une sculpture. (Arp, 1966, p. 323) ${ }^{20}$ 
Pour conclure, la créativité et l'image, lorsque la forme nous informe, sont des grands médiateurs de connexion avec l'environnement. A travers la multiplication des images et l'exercice de la pensée analogique ${ }^{21}$, on intercepte un univers de significations. Se mettre en état de disponibilité pour accueillir les images et rentrer dans ce monde d'allégories, c'est faire une incursion dans une sorte d'organisation parallèle du sens, un monde de synchronicité où les images se succèdent, se génèrent les unes les autres. Les sons, les couleurs, les lignes et les formes nous permettent d'accéder aux symboles. Le mythe, la condensation, les déplacements et les associations d'idées dessinent d'autres liens... Monde de résonances, accord invisible dont parle Matisse à propos de sa mise en condition pour peindre ? Pour cet artiste, « l'esprit, flottant dans la rêverie, perçoit peu à peu, sans y prendre garde, d'autres rapports entre les choses, une sorte d'accord invisible qui existerait entre les formes, les couleurs, et les ferait soudain résonner toutes ensemble ».

L'art est ainsi un vecteur de liens avec le monde qui peut nous transcender et nous toucher à plusieurs niveaux de compréhension, nous renvoyant à de multiples dimensions, comme peut le faire le chamane. Sollicitant une pensée moins binaire ou plus holistique, il nous fait vivre une expérience augmentée, car la forme joue son jeu en relief et parle... L'art est support de prises de conscience et d'ouverture de la porte du cerveau intuitif. Dans ce sens-là, il prolonge la réflexion.

L'art colorie le monde, le réenchante, car il transforme la vision fonctionnelle $d u$ réel en lui redonnant du sens et du volume, en nous reliant à l'environnement dans une relation « vivante » et habitée. Il n'est donc pas seulement subjectif mais peutêtre outil de cognition. Les couleurs et l'image appellent à des résonnances transversales. Ce qui n'est pas arrêté et fixé, précisément le «flou » artistique, appelle à des liens de correspondance. Ces «tentacules» de l'imaginaire renseignent sur les espérances, nous permettent de rattraper ce que l'on ne sait pas consciemment. C'est ainsi que l'œuvre se fait miroir pour agir comme révélateur de notre lien intrinsèque au réel et que l'imaginaire nous précède pour nous permettre de transformer notre rapport au monde. (Planche, à paraitre 2018, p. 70)

Ainsi qu'un coucher de soleil qui vous reconnecte avec le ciel, les couchers de peinture vous entrainent à la porte des énergies invisibles de la dimension vibratoire des couleurs et des formes. Non seulement, la forme est informante et s'adresse à d'autres véhicules que la rationalité, mais la créativité est un grand vecteur de connexion avec les objets du monde. Elle nous relie à notre environnement.

\section{Pistes d'atelier : Jouer avec la classification de Descola pour une approche ethnopédagogique ${ }^{22}$}

Signalons d'entrée de jeu qu'il ne s'agit pas ici d'une fiche pratique, mais d'un exemple d'articulation entre les concepts et leur mise en œuvre pédagogique.

Lors d'une balade poétique ou de séquences en alternance d'approches - avec des enfants ou des adultes - nous proposons comme consigne de prendre le point de vue de l'animisme et du naturalisme ${ }^{23}$, selon la classification de Descola $(2005$, p. 323) et de les mettre en perspective (Tableau 1). 
Tableau 1. Comparaison entre l'animisme et le naturalisme : extrapolation à partir de la classification de Philippe Descola

\begin{tabular}{|l|l|}
\hline ANIMISME & NATURALISME \\
\hline $\begin{array}{l}\text { Fond } \rightarrow \text { semblable = énergie de vie, } \\
\text { capacité d'émettre un point de vue sur le } \\
\text { monde }\end{array}$ & $\begin{array}{l}\text { Fond } \rightarrow \text { différent = 1'humain pense, } \\
\text { s'échappe de son déterminisme naturel } \\
\text { et a une capacité culturelle de } \\
\text { Forme } \rightarrow \text { différente = grenouille, humain, } \\
\text { arbre restent animés par l'énergie de vie. La } \\
\text { forme crée un point de vue différent : la } \\
\text { grenouille saute, rebondit. Son Umwelt d'intentions. Les autres } \\
\text { (mode de vie et d'être) est étroitement lié à } \\
\text { son outillage qui correspond à une } \\
\text { perception différente du monde dans laquelle } \\
\text { nous allons essayer de nous glisser grâce àntention propre, } \\
\text { sont mus par un mécanisme biologique. } \\
\text { Forme } \rightarrow \text { extériorité semblable, } \\
\text { contour de la fabrication du vivant, } \\
\text { matière biologique, hors de la raison } \\
\text { d'être et du sens. Grenouille, humain, } \\
\text { arbre restent faits de la même matière } \\
\text { chimique. }\end{array}$ \\
\hline
\end{tabular}

L'imaginaire opérationnel permet de quitter sa hauteur, pour se mettre dans la peau de l'animal ou dans le point de vue de la plante ou de l'arbre, pour expérimenter d'autres façons d'appréhender le monde. Nous épouserons le point de vue de l'arbre ou bien de la plante dans sa relation à l'insecte, ou encore celui de l'animal : l'oiseau, le poisson qui vit sous l'eau et est témoin de ce monde a priori dissimulé à l'être humain, jusqu'à celui du nuage qui observe tout depuis le ciel. L'animiste va redonner une âme aux choses, en particulier aux éléments naturels (ou va "remettre des esprits dans la forêt », soit des liens et des symboles) ; il va les faire « parler » et « penser. » Un arbre, un objet peuvent être animés, mais ne pas avoir la même forme. Une pierre, un humain, une plante, un animal... un nuage : chacun peut "penser ", parler, être. Le fond est semblable dans le sens " d'animé », tandis que la forme est différente.

41 A l'image de l'animiste, nous chercherons à atteindre les secrets qui sont de l'autre côté du visage humain: du côté du paradigme de la nature, de l'intention des autres existants. Nous adopterons des points de vue animaux: celui de la grenouille, du renard, de l'oiseau..., en nous appuyant sur notre imaginaire, dans un va-et-vient avec des données scientifiques éthologiques: équipement de l'animal (vision, odorat, système de défense), alimentation, niche écologique, etc. Ces données serviront d'appui pour se mettre en situation. Aussi, pour rentrer dans la peau de l'animal, il faut effectuer un va-et-vient entre les sensations qu'on éprouve en tentant d'adopter sa posture (par exemple le vol d'un oiseau) et des informations éthologiques disponibles (le blaireau a un odorat 700 fois plus développé que l'Homme!). "Pour connaitre le bambou, il faut se mettre dans la peau du bambou », dit la pensée asiatique.

Nous cherchons avec le naturalisme à percevoir comment l'animal est constitué. Avec l'animisme, nous essayons de percevoir pourquoi il fonctionne, pour tendre vers quel dessein. Il s'agit donc d'observer ces autres existants "de l'intérieur", selon leur paradigme a priori imperceptible à l'humain: par exemple, la plante cherche le jour, s'étire et s'étend vers ces points de lumière. L'appareillage de la grenouille est constitué d'yeux exorbités pour mieux voir à la surface de l'eau. Au lieu de disséquer la grenouille, nous allons essayer de l'imiter. Mais, pour jouer « le point de vue de », nous aurons forcément recours à l'imaginaire, car le saisissement de cet autre imperceptible ne peut se faire que par le canal de l'équipement humain: la parole, le corps, l'imaginaire... et l'art ! 
43 L'éthologue Pierre Clément met en évidence la spécificité visuelle de chaque animal, qui oriente le paradigme particulier de sa perception sensorielle (Clément, 1999, p. 20-25). Allons-nous adopter, avec la grenouille, le point de vue d'un peintre pointilliste? Ou bien, avec certains insectes ou mammifères, le point de vue monochrome, imitant en cela le peintre dans sa période bleue ou rouge?

L'important dans cet atelier est de mettre en jeu un échange avec le milieu, pour un temps donné, la «rectification» scientifique pouvant se faire a posteriori, avec une envie de découvrir stimulée. Il est ainsi intéressant d'observer les animaux disponibles, dans le silence et l'attention lente, comme le fait le peintre naturaliste ou comme le danse l'Inuit. Nous proposons finalement de mettre en perspective le point de vue naturaliste avec le matériel «animiste " recueilli à travers la posture du poète. Nous confronterons la réalité en aval des productions artistiques et imaginaires, afin de rebondir sur des notions de biologie morpho-fonctionnelle et d'éthologie.

Sur un plan pédagogique, il s'agit tout d'abord de vivre une expérience afin de favoriser une création et/ou l'envie d'apprendre. Par ailleurs, l'atelier va inverser les paradigmes pour appréhender l'exercice poétique, pour assimiler la notion de diversité du vivant, ainsi que pour prendre conscience de la diversité des cultures. D'une part, l'atelier est un levier de créativité pour l'art : on observe que s'abreuver au lait de la nature fait le tiers avec les présupposés sur l'esthétique artistique pour aboutir à une production poétique plus libre, imaginative et personnelle. Les écrivants se délestent de leurs blocages car la nature leur donne la main pour «s'écrier » avant de penser au «bienécrire ». D'autre part, l'atelier est un levier d'intérêt pour la science : l'appropriation créative, l'expérience sensorielle et récréative motivent les apprentissages scientifiques et l'envie d'en savoir plus sur le sujet.

Par ailleurs, l'atelier permet de se décentrer de sa posture d'être savant (soit de sa posture naturaliste) pour rentrer dans le milieu, «s'implicare» au sens de Dominique Cottereau, c'est-à-dire d'abord se "naturer", afin de s'ouvrir à de nouveaux apprentissages. Ce décentrement répond à un triple objectif. Il familiarise avec l'inversion poétique - ce n'est pas le vent qui agite les feuilles, mais l'arbre qui murmure - ainsi qu'avec la notion de biologie morpho-fonctionnelle qui nous montre que les êtres vivants évoluent dans des réalités perceptives différentes afférentes à leurs organes biologiques. Tout en le décentrant par rapport à ses références culturelles, l'atelier permet à l'apprenant d'intégrer des notions de classifications ethnologiques du rapport des sociétés à la nature.

\section{BIBLIOGRAPHIE}

Archives INA. (1959). L'atelier de Jean Arp, lieu de création. http://fresques.ina.fr/jalons/fichemedia/InaEdu05343/l-atelier-de-jean-arp-lieu-de-creation.html

Arp, J. (1966). Jours effeuillés : poèmes, essais, souvenirs, 1920-1965. Paris : Éditions Gallimard.

Aurégan, P. (2014). Jean Malaurie, une introduction, Paris : Agora, Pocket. 
Bastide, R. (1965). La pensée obscure et confuse. Le Monde chrétien, 75-76, p. 137-156.

Clément P. (1999). Autant d'espèces, autant de mondes! Des mondes animaux. Science et Avenir, 119 (7-8), p. 20-25.

Cottereau, D. et Bachelart, D. (2003). Groupe de recherche en Éco-formation. Origines du concept, Universités de Paris 8 et de Tours-François Rabelais. URL : http://www.barbier-rd.nom.fr/ GREFDCottereau2002.html.

Descola, P. (2005). Par-delà nature et culture. Paris : Gallimard.

Etiembre, Y. (2015). L'homme qui parlait avec les pierres. Jean Malaurie. Sciences et poétique de l'Arctique. http://agoras.typepad.fr/regard_eloigne/2015/06/lhomme-qui-parlait-avec-lespierres-jean-malaurie-sciences-et-poetique-de-larctique-2.html.

Feyerabend, P. (1989). Adieu la raison. Paris : Seuil.

Laplantine, F. (1996). La description ethnographique. Paris : Nathan.

Laplantine, F. (2005). Le social et le sensible: Introduction à une anthropologie modale. Paris : Téraèdre.

Lenoir, F. (2015). La puissance de la joie, Paris : Fayard.

Les abstractions I. (1986). La diffusion des Abstractions, Hommage à Jean Laude, Centre Interdisciplinaire d'Études et de Recherches sur l'Expression Contemporaine, Université de SaintÉtienne, Travaux XL VIII, p. 61.

Malaurie, J. (2002). Pas à pas avec vers les Inuit. Dans Séminaire de Jean Malaurie 2000-2001, De la vérité en Ethnologie. (p. 107-148). Paris : Economica.

Malaurie, J. (2013). Les derniers rois de Thulé, $5^{\mathrm{ème}}$ édition, Paris : Plon.

Malaurie, J. (2015). Lettre à un Inuit de 2022. Paris : Fayard.

Morin, E. (1997). Amour, poésie, sagesse. Paris : Seuil.

Pardo, T. (2002). Héritages Buissonniers. Éléments d'ethnopédagogie pour l'éducation relative à

l'environnement. La Caunette : Babio.

Pineau, G. (1992). De l'air ! Essai sur l'écoformation. Paris : L'Harmattan.

Planche, E. (1989). Coutelier et/ou thiernois, essai de définition d'une identité culturelle, In Hadjadj, D. (dir.). Pays de Thiers, le Regard et la mémoire. (p. 177-196). Clermont-Ferrand : Institut d'Études du Massif Central, Université Blaise Pascal.

Planche, E. (2008). Le Rhône, la mallette des merveilles du fleuve. Outil magique et pédagogique pour un lien entre les jeunes riverains. La Mulatière : Science et Art.

Planche, E. (2008). Balade poétique. Dans Le Rhône, la mallette des merveilles du fleuve. La Mulatière : SeA, Science et Art.

Planche, E. (2011). La balade poétique ou la posture de l'animiste. Dans Réseau École et Nature. Regards d'ici et d'ailleurs, Quand l'éducation à l'environnement explore les liens que tout peuple tisse avec la nature. (p. 64-65). Montpellier : École et Nature.

Planche, E. (2011). Le rapport de l'Homme à son environnement et la notion de sujet. Dans Giordan, A., Martinand, J.-L., et Eastes, R.-E. (Dir.). L'idée de nature dans la médiation et l'éducation scientifique. Actes des $31^{e}$ Journées internationales sur la communication, l'éducation et la culture scientifiques, techniques et industrielles. (p. 213-226). Paris : Traces et ENS Cachan. http:// artheque.enscachan.fr/items/show/419 
Planche, E. (2018). Eduquer à l'environnement par l'approche sensible. Art, ethnologie et écologie. Lyon : Editions Chronique sociale.

Planche, E. (Texte inédit 2017, à paraître 2019). « Réveiller la force. Cinq leçons pour « réanimiser » les peuples du Nord ». Dans Malaurie, J., Arctica. Euvres II. Tchoukotka 1990, Paris, CNRS Éditions.

Platon, CEuvres complètes, tome $4,3^{\text {ème }}$ partie, Phèdre, texte établi et traduit par Léon Robin (1970). Paris : Les Belles Lettres, p. 32-33.

Rimbaud, A. (1975). Lettres du voyant (13 et 15 mai 1871). Genève : Librairie Droz.

Sauvé, L. (1998). L'éducation relative à l'environnement - Entre modernité et postmodernité : Les propositions du développement durable et de l'avenir viable. Dans Jarnet, A., Jickling, B., Sauvé, L., Wals, A. et Clarkin, P. (dir.). A colloquium on the future of environmental education in a postmodern world? Proceedings of an on-line colloquium held on October 19th 1998, p. 57-70.

Sorman, G. (1989). Les vrais penseurs de notre temps. Paris : Fayard, 1989.

Tadié, M. (2002). Perceptions extra-sensorielles des populations primitives. Dans Malaurie, J., S éminaire de Jean Malaurie 2000-2001, De la vérité en Ethnologie, Paris : Economica, p. 31-53.

Terrasson, F. (1997). La peur de la nature. Paris : Sang de la Terre.

\section{NOTES DE FIN}

1. www.science-et-art.com

2. Pour nous, il s'agit de l'environnement "au sens large ", évoquant ainsi une dimension plus complète qui inclut le fait de se relier aux murs de la ville par l'émotion et la mémoire, de se relier aux objets du monde par le symbole qui fructifie, tisse des liens de résonances entre couleurs et formes sur un autre plan que la logique classificatoire. Rappelons néanmoins que, pour Lucie Sauvé, « il n'existe pas une définition consensuelle de l'environnement. Il s'agit d'une réalité culturellement et contextuellement déterminée, socialement construite. » Récupéré de http://assises-ere.be/4jours/Lucie-Sauve.php.

3. Claude Levi-Strauss précise bien que la "pensée sauvage " n'est pas la pensée des sauvages, mais une forme de raisonnement fécond et universel qui peut s'exprimer dans chaque société (Sorman, 1989, p. 128).

4. Le comté, fromage de lait cru de vache, se présente en meules affinées dans une cave.

5. Intervention de Laplantine, F., Conférence à l'Université catholique de Lyon, 10 mars, 2016.

6. Malgré les nuances de la pensée d'un philosophe qui restent plus subtiles que sa trace.

7. Lire les analyses de Sauvé (1998).

8. Il s'agit bien ici de lier - dans une systémie de liens ancrés au local, au sol - les savoir-faire, par exemple fabriquer un couteau, avec les savoir-être, par exemple celui de l'émouleur, joyeux, buveur, libre : exemplerations sorcellaires, qui cherche à l' fort ne serait pas un jaguar déguisé en homme et si cet oiseau qui pépie sur l'exemplerations sorcellaires, qui cherche à l' fort ne serait pas un jaguar déguisé en homme et si cet oiseau qui pépie sur l'son geste s'inscrit dans une dimension vie/mort qui sacralise son corps de métier, tout en forgeant une attitude de défi, car il risque sa vie avec l'éclatement fréquent de la meule ; en même temps, il représente la noblesse du geste qui fait vivre la ville par le couteau, puisque dans la chaîne du couteau, c'est l'émouleur qui fait son tranchant, soit sa raison d'être. (Planche, 1989)

9. Qui pourrait s'apparenter à un chaman de nos contrées.

10. Jean Malaurie explique comment les esquimaux, pour ajuster leurs structures sociales et leurs actions en fonction du climat, examinent avec attention les moindres signes et mouvements de 
leur environnement, jusqu'au brouillard dégagé par l'haleine des chiens (Malaurie, 2013, p. 159-160).

11. Edgar Morin affirme la coexistence structurelle chez l'humain, de deux formes de langage correspondant à deux états : «L'état premier, qu'on peut appeler prosaïque et l'état que l'on peut justement appeler "état second", l'état poétique » (Morin, 1997, p. 39-40).

12. Rencontre organisée par la Région Rhône-Alpes : Biens Publics mondiaux : Forêt, biodiversité et peuples autochtones. Le Peuple originaire kichwa de Sarayaku, 26 septembre 2012, Conseil régional Rhône-Alpes.

13. Pour nous, la classification de Descola $(2005$, p. 323) reste un idéal type opératoire utile à l'analyse : en réalité, les modèles se manifestent et s'interpénètrent plus ou moins dans chaque société. Ce qui nous intéresse ici, c'est de montrer comment la notion de naturalisme renvoyant à une extériorité semblable des corps vivants évoque l'idée d'une matière non intentionnelle, alors que l'animisme propose une intention pour chaque élément du vivant; nous rejoignons ainsi Malaurie (2002, p. 120) qui parle d'un ordre de la nature et d'une nature en projet.

14. Pour une étude plus complexe et complète, voir Descola (2005, p. 232)

15. Voir le schéma d'Édith Planche (2011): « Le modèle occidental et le modèle animiste au sens générique ", dans Le rapport de l'Homme à son environnement et la notion de sujet, op. cit, p. 217.

16. Voir : https://www.tate.org.uk/art/artworks/doig-reflection-what-does-your-soul-look-likep11544

17. Terrasson, F. (2002). Le développement durable : réalité ou beau discours ? Conférence à la Maison Rhodanienne de l'Environnement, Lyon.

18. Voir par exemple Une balade SeA avec l'école Condorcet de Valence: http:// culturebox.francetvinfo.fr/t.

19. Pour un atelier afférent, voir Troisième leçon, dans Planche, E., «Réveiller la Force ", Dans Malaurie, J., Arctica. CEuvres II. Tchoukotka 1990, Paris, CNRS Éditions, à paraître en 2019, op. cit.

20. Pour un atelier autour de Jean Arp, voir "Collecte Nature et Culture » dans l'ouvrage Eduquer à l'environnement par l'approche sensible (Planche, 2018, p. 276).

21. Le terme renvoie ici à un plan artistique et non pas ethnologique. J'utilise la pensée analogique à travers ma pratique de l'aquarelle, au niveau de la dynamique des couleurs et des formes, en apposant par exemple une tache rouge qui résonne avec ce toit rouge.

22. Pour une définition du concept, consulter l'ouvrage de Thierry Pardo (2002).

23. Pour un atelier plus complet incluant le "totémisme", se référer à l'ouvrage Éduquer à l'environnement par le sensible (Planche, 2018, p. 375-378).

\section{RÉSUMÉS}

Le modèle de la "pensée rationnelle » nous invite à exclure les sens et à nous séparer du réel, donc de la nature, pour mieux l'analyser. L'individu moderne est séparé non seulement de la nature, mais des objets du monde, car il envisage son environnement de manière fonctionnelle. La poésie et l'art de manière générique, permettent de laisser vivre une forme de "pensée sauvage ", " matrice secondaire » de nos sociétés contemporaines. Nous allons montrer comment se mettre à l'écoute de la résonance des couleurs et des formes, comme l'artiste, ou se mettre à l'écoute de sa propre résonance au monde, comme le poète, nous permet de tisser des liens, de lancer des tentacules pour mieux entrer en relation avec l'environnement. 
Rational thinking encourages us to repress our sensitivity and to separates ourselves from materiality - thus from nature - for a better understanding of reality. Because he/she views his environment in a utilitarian way, the individual in modern society is disconnected not only from nature but also from objects of the world. Poetry and art give birth to a form of "savage mind", submatrix of our contemporary society. This process of breeding images helps developing relationships with the environment in its broadest meaning.

\section{INDEX}

Mots-clés : art, environnement, forme, symbole, pensée sauvage, poésie

Keywords : art, environment, form, symbol, wild thinking, poetry

\section{AUTEUR}

\section{EDITH PLANCHE}

Ethnologue, chercheure associée au laboratoire EVS (UMR 5600 CNRS) de l'Université de Lyon, Edith Planche a publié dans des ouvrages collectifs en sciences humaines, ainsi qu'un ouvrage ethno-poétique. Elle a fondé SEA, Science et Art, structure d'éducation à l'environnement par la culture, s'adressant à tous publics et partenaires de collectivités territoriales. Elle est auteure et illustratrice de nombreux supports associant fond et forme, rassemblés dans l'outil pédagogique Le Rhône, la mallette des merveilles du fleuve. 